\title{
PVA Nanofibers Containing Ofloxacin-Cyclodextrin Inclusion Complex: Improve Optical Stability of Ofloxacin
}

\author{
Mohammad Mehrizi ${ }^{1}$, Sahar Amiri ${ }^{*}$, Seyed Hajir Bahrami ${ }^{2}$ \\ ${ }^{1}$ Department of Polymer Engineering, Science and Research Branch, Islamic Azad University, Tehran, Iran \\ ${ }^{2}$ Department of Textile Engineering, Amirkabir University of Tehran, Tehran, Iran \\ Email: *s.amiri@srbiau.ac.ir
}

How to cite this paper: Mehrizi, M., Amiri, S. and Bahrami, S.H. (2019) PVA Nanofibers Containing Ofloxacin-Cyclodextrin Inclusion Complex: Improve Optical Stability of Ofloxacin. Open Journal of Organic Polymer Materials, 9, 29-46.

https://doi.org/10.4236/ojopm.2019.92002

Received: February 17, 2019

Accepted: April 7, 2019

Published: April 10, 2019

Copyright () 2019 by author(s) and Scientific Research Publishing Inc. This work is licensed under the Creative Commons Attribution International License (CC BY 4.0).

http://creativecommons.org/licenses/by/4.0/

\begin{abstract}
Ofloxacin is an antibiotic with a wide range of activity against bacterial infections, but due to the high potential for toxicity when exposed to light, resolving this problem and further stabilizing the drug are among the posed challenges. Inclusion complex formation between $\alpha$-cyclodextrin ( $\alpha$-CD), ofloxacin (OFL) and polyethylene glycol (PEG) was prepared via two methods to produce nanocontainers with desirable stability. The effect of PEG as compatible solubilizing agent and mixing condition (in ultrasonic bath) were investigated in formation of an inclusion complex between $\alpha$-CD/OFL. Obtained complexes were examined by FTIR, H-NMR, SEM, EDX and UV which indicated the formation of an inclusion complex between $\alpha$-CD/OFL, in turn, is a mixture of the cage and channel structures. Differences between ${ }^{1} \mathrm{H}$-NMR, FTIR and XRD spectra of OFL, CDs and inclusion complex indicated the formation of $\alpha$-CD/OFL and supramolecular containers in solid phase. These inclusion complexes loaded in PVA-based nanofibers for smart nanofibers with controlled release manner and higher stability of OFL. Obtained nanofiber showed that nanofibers containing CDs/OFL under sonic energy containing higher degree of OFL.
\end{abstract}

\section{Keywords}

Supramolecular Containers, $\alpha$-Cyclodextrin, Ofloxacin, Optical Stability, Electrospinning

\section{Introduction}

Today, the effects of light and resulting toxicity on many medicines have been established [1] [2]. Ofloxacin is no exception and has high optical sensitivity. 
Due to the exposure to direct light, in addition to reducing the amount of drug activity, the substances resulting from photodegradation are highly reactive and toxic and their consumption will lead to serious problems for the patient. Therefore, it is essential to resolve these problems [1] [2]. Tiefenbacher et al. (1994), investigated that the photodegradation of fluoroquinolones, such as ciprofloxacin, ofloxacin and fleroxacin by ultraviolet irradiation and natural room light in liquid medium [3]. In the most optimistic case, it can be said that the efficacy of the drug is reduced to some extent, but this is often not the case, and the side effects that appear due to the formation of degradation-induced substances are very dangerous and important (Scheme 1) [3].

All research activities for increasing the shelf life and preventing the degradation of the drug during the production and maintenance stages are divided into two general parts: Packaging and drug modification. The drugs, including ofloxacin, inherently have disadvantages such as low solubility, low bioavailability, low stability, and so on, which provides the basis for drug modification [4].

One method to improve stability and bioavailability of poorly water-soluble drugs is formation of an inclusion complex between drugs and Cyclodextrins. Cyclodextrins (CDs) are cyclic oligosaccharides with a unique structure consisting of several glucopyranose units and are often described as truncated cone-shaped structures with a hydrophilic exterior surface and a hydrophobic interior cavity. By formation of the inclusion complex, the interior cavity of cyclodextrin has a profound effect on the chemical-physical properties of guest molecules which increase the effective properties of guest molecules, because they are temporarily bound or they are caged in the host cavity [5] [6].

Formation of inclusion complex may change guest molecules properties such as increasing the solubility of ofloxacin in aqueous medium, thermal stability, eliminating the bad odor of the drug, controlled release, etc. Another important feature of cyclodextrin is the optical stability of guest molecules within the

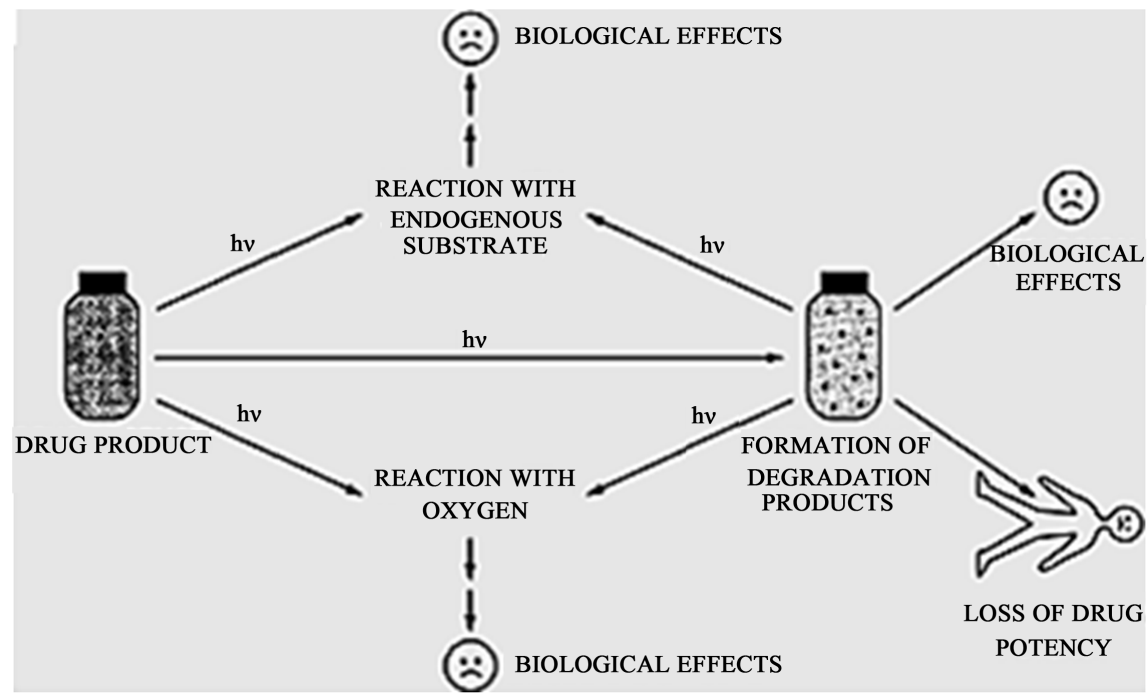

Scheme 1. Effect of light on drug toxicity [3]. 
hydrophobic cavity of cyclodextrins [5] [6]. CDs can encapsulate the hydrophobic substances into their cavities and prevent the direct contact of substances with light. In fact, if using $\mathrm{CD}$, we can cover some parts of ofloxacin which show light sensitivity and are degraded by contact with light, then the degradation rate will be significantly reduced and the drug exposed to light will maintain its integrity and efficacy for a longer time [5] [6].

In the present research, formation of an inclusion complex of $\alpha$-cyclodextrin $(\alpha-\mathrm{CD})$ and ofloxacin (OFL) in ultrasonic bath and PEG effect were evaluated. Obtained complexes were analyzed by FTIR, H-NMR, SEM, EDX and UV which indicated confirmation of nanocapsules of OFL/CDs formation under various conditions. Obtained nanocontainers were loaded into Poly(vinyl alcohol) (PVA) via electrospinning due to high surface area, high porosity and compatibility by functionalizing additives for a wide range application such as smart drug delivery systems or improve stability of drugs. OFL/CD act as antibacterial agent and released with a controlled manner. In electrospinning of PVA with OFL/CD complex, an efficient amount of nanocontainers added to PVA solution, which can be used for synthesis of nanofibers with specific properties for specific application such as wound dressing, drug delivery systems, cell culture and antibacterial cloths. SEM and EDX were used to investigate structure and morphology of obtained nanofibers and the release of OFL from PVA nanofibers was determined by UV-Visible. Antimicrobial activity of nanofibers was tested against Escherichia coli (E. coli) and Staphylococcus aureus (S. aureus) representing Gram-negative and Gram-positive bacteria, respectively.

\section{Methods}

Poly(vinyl alcohol) (PVA, Mw $=72,000$, Merck, $=98 \%), \alpha$-cyclodextrin $(\alpha$-CD, Applichem, =98\%), ofloxacin (OFL, Aldrich, >98\%), citric acid (Merck, >98\%) and ethanol (EtOH) (Merck, >98\%) were used in absence of further purification [6] [7] [8] [9].

\subsection{Characterization}

Bruker DRX 500 spectrometer $(500 \mathrm{MHz})$ was used to record hydrogen nuclear magnetic resonance ( ${ }^{1} \mathrm{H}$-NMR) spectra in DMSO, at room temperature, 3.080 ppm, $500 \mathrm{MHz}$. Infrared spectra were recorded with a Bruker IFS 48 FTIR spectrometer. To study the diffraction patterns of cyclodextrin/OFL crystals at ambient conditions X-ray diffraction (XRD) was considered; an Xpert Philips diffractometer (USA) with nickel filtered $\mathrm{Cu} \mathrm{K} \alpha$ radiation was applied. Data were collected at a rate of $2^{\circ} \theta$ min over the $2 \theta=5^{\circ} \approx 60^{\circ}$ range. Scanning electron microscopy (SEM), Philips XL30 (Poland), was used to shed light complexes structures. UV-Vis spectrophotometer (Shimadzu UV-1650 PC) at given adsorptions was used in phosphate buffer. The antimicrobial properties of the nanofibers were investigated by bacterial growth against Escherichia coli (E. coli) and Staphylococcus aureus ( $S$. aureus) representing Gram-negative and Gram-positive bacteria, respectively [6] [7] [8]. 


\subsection{Inclusion Complex Formation of CD/OFL under Sonic Energy}

Inclusion complex formation of $\mathrm{CD} / \mathrm{OFL}$ under sonic energy upon adding an OFL solution in ethanol ( $5.0 \mathrm{ml}$ of $14.5 \mathrm{wt} \%)$ to $\alpha$-CD solution in ethanol $(5.0 \mathrm{ml}$ of $5.0 \mathrm{wt} \%)$. The suspension was sonicated for $30 \mathrm{~min}$ while the temperature of solution was kept on $25^{\circ} \mathrm{C}$ using ice-water bath, then stay at room temperature $\left(25^{\circ} \mathrm{C}\right)$ for further 24 hours. In this research Q500, Ultrasonic Probe Sonicator was used with 500-watt power for nanoparticle dispersion and homogenization.

After preparing, the obtained complexes were washed with ethyl ether to remove any excess of free OFL and CD. At the end of the reaction, the final solution was recovered by filtration and it was dried in a vacuum oven at $50^{\circ} \mathrm{C}$ in an oven for $24 \mathrm{~h}$. The dried complex was characterized by XRD, SEM, EDX, FTIR and ${ }^{1} \mathrm{H}-\mathrm{NMR}$ [6] [7] [8].

To test the optical stability and to evaluate the effect of UVA light on the complexes, each of the prepared complexes in is exposed to the UVA light along with the pure OFL to the same weight for 13 days [6]. For this purpose, an 8-watt light bulb and an aluminum foil-insulated wooden chamber are used to prevent the light from entering and exiting. Optical stability of OFL against light is one of the important goals in preventing the production of toxic active products. After a specified time from the presence of pure drug and the complexes prepared by exposure to UVA light, all three samples were measured at identical weights to determine the amount of optical degradation in the UV-Visible device.

\subsection{Electrospinning}

Electrospinning was carried out by preparing PVA and IC solutions, PVA solutions $(9 \% \mathrm{w} / \mathrm{w})$ in distilled water. After formation, a homogenous solution, $0.037 \mathrm{~g}$ $\mathrm{CDs} / \mathrm{OFL}$ inclusion complexes and $10 \%(\mathrm{w} / \mathrm{w})$ citric acid (compared to the PVA weight) were added to the obtained PVA solution [7] [8] [9].

The solution was placed on a magnetic stirrer in ambient temperature at 250 rpm for 16 hours. The solution was drawn into a $5-\mathrm{mL}$ glass syringe with a $30 \mathrm{G}$ needle and electrospinning was carried out with voltage $15 \mathrm{kV}, 20 \mathrm{~cm}$ for distance between the needle tip and the collector (aluminum foil covered collector) and flow rate of $3 \mu \mathrm{l} / \mathrm{min}$. Electrospinning experiments were carried out in an enclosed box at $23^{\circ} \mathrm{C}-26^{\circ} \mathrm{C}$ and $18 \%$ relative humidity [7] [8] [9] [10]. After performing the electrospinning, the obtained nanofibers were placed in an oven for 24 hours at $45^{\circ} \mathrm{C}$ to eliminate the resulting moisture. This will improve the stability and consistency of nanofiber and reduce the weight loss of nanofibers in aqueous media. The fibers are immersed in a $1 \mathrm{M} \mathrm{NaOH}$ solution and placed at ambient temperature to be dry. By doing this, the process of cross-linking is complete, preventing the full dissolution of PVA in water [6] [7] [8] [9].

\subsection{Anti-Bacterial Efficiencies}

Anti-bacterial activities of the coatings were evaluated against Escherichia coli ( $E$. coli, ATCC25922) and Staphylococcus aureus (S. aureus, ATCC25923) representing 
Gram-negative bacteria and Gram-positive bacteria, respectively. Anti-bacterial efficacies were determined according to AATCC 100-2004 test method. Anti-bacterial activities were observed when the bacteria inoculum was surrounded by a clear zone of growth inhibition, and evaluated by determining both the diameter of the bacteria colony and the diameter of the inhibition halo [6] [7].

\section{Results and Discussions}

\subsection{Stoichiometry's}

The complex formation of $\alpha$-CD with OFL was studied quantitatively. The amount of the complex formed increases with an increase in the amount of OFL added to the aqueous solution of CDs. The amount of the complex showed similar values even if excess amounts of OFL were used; this indicates the stoichiometric complexation. The continuous variation plot for the formation of the complex between CDs and OFL is at the maximum level at 0.50 (monomer unit:CD = 1:1) stoichiometry (Figure 1 ). This result suggests that 1 units were bound in each CD cavity.

The structures of obtained supramolecular nanocontainers of OFL/a-CD were studied with various methods (FTIR, XRD and SEM) in our previous work [6]. Also, nanocontainers were characterized with ${ }^{1} \mathrm{H}-\mathrm{NMR}$ which is one of the most useful methods for detecting the formation of an inclusion complex. The ${ }^{1} \mathrm{H}$-NMR spectrum of $\alpha$-CD, OFL, and obtained inclusion complexes is shown in Figure 2. All signals of the ${ }^{1} \mathrm{H}-\mathrm{NMR}$ spectra were assigned to their corresponding monomers and it can be declared the synthesis of OFL/ $\alpha$-CD [6] [7] [8] [9].

The entrapment of the guest molecule in the $\alpha$-CD hydrophobic cavity is confirmed with chemical shift of interior protons (H-3, H-5 and H-6) of CDs significantly compared to exterior protons $(\mathrm{H}-2$ and $\mathrm{H}-4)$. Interior protons of $\mathrm{CDs}$

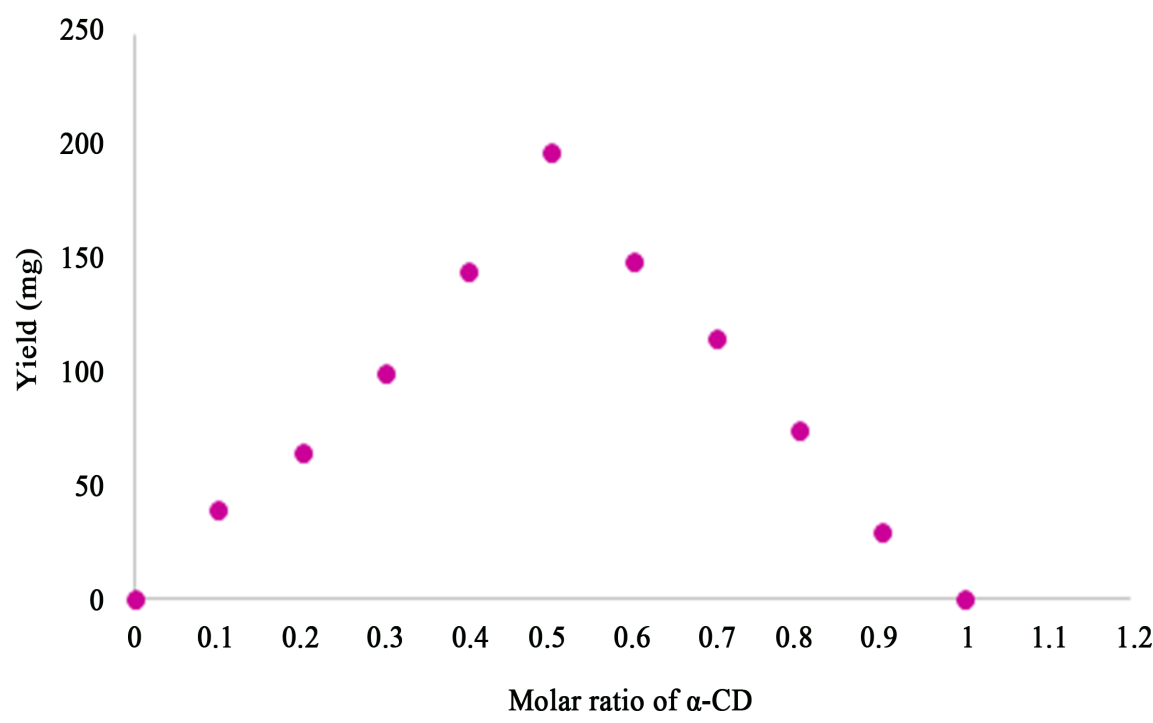

Figure 1. Continuous variation plot for complex formation between CD and OFL under sonic energy. 


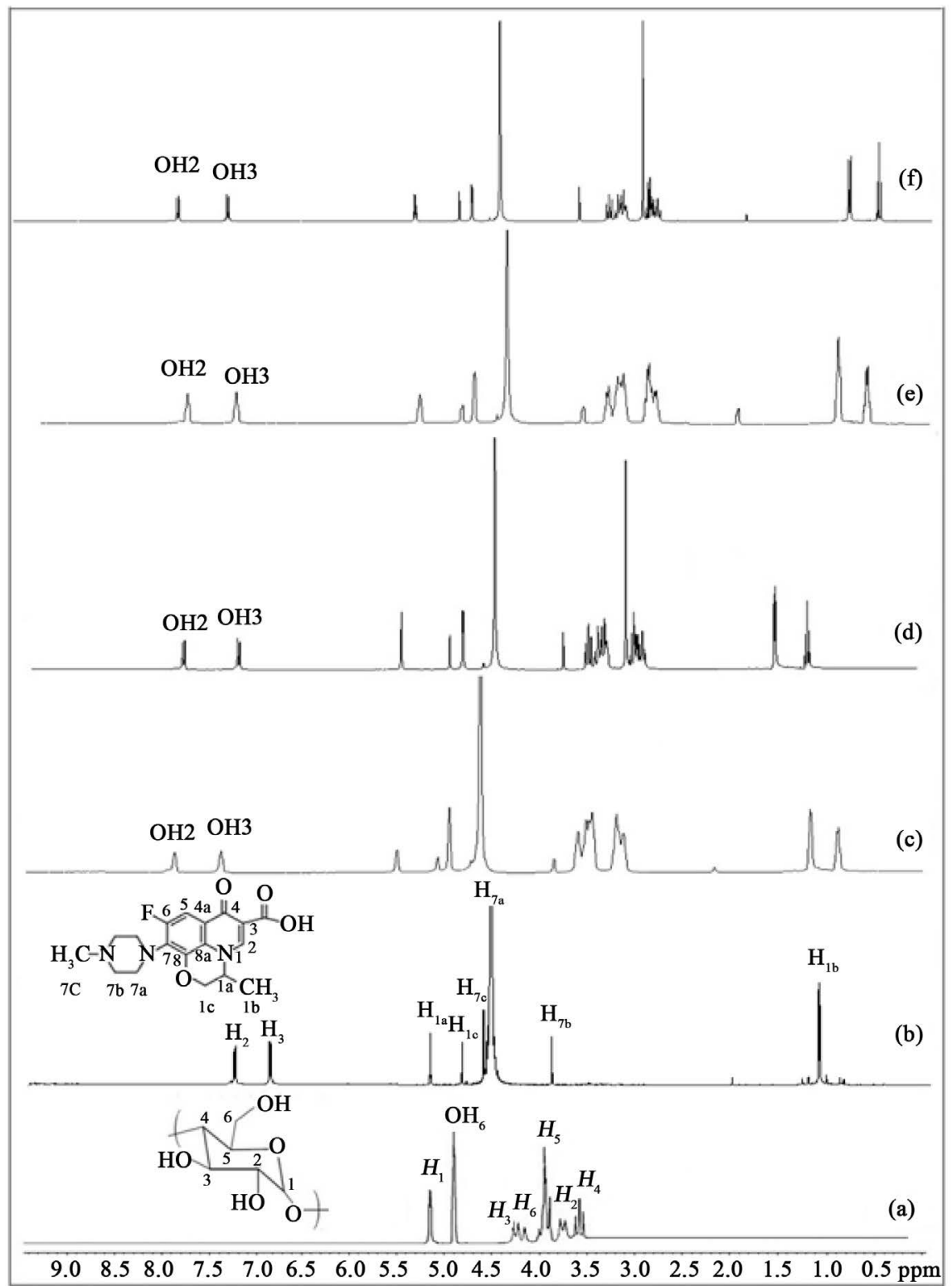

Figure 2. ${ }^{1} \mathrm{H}$-NMR Spectra of (a) Pure $\alpha$-CD; (b) Pure OFL; (c) OFL/ $\alpha$-CD (before degradation); (d) OFL/ $\alpha$-CD (after degradation); (e) OFL/ $\alpha$-CD/PEG (before degradation) and (f) OFL $\alpha$-CD/PEG (after degradation).

took part in the interaction due to the deep penetration of the OFL molecule into the $\mathrm{CD}$ cavity and the site of interaction could be the secondary alcoholic group at $\mathrm{H}-3$. On the other hand, it can be seen that $\mathrm{H}-3, \mathrm{H}-5$ and $\mathrm{H}-6$ experience the highest shift, which is due to the decrease in the freedom of action and the rotation of the OFL molecule due to the placement interior the $\alpha$-CD cavity. In the other case, $\mathrm{H}_{7-\mathrm{b}}, \mathrm{H}_{7-\mathrm{a}}, \mathrm{H}_{7-\mathrm{c}}$, and $\mathrm{H}_{1-\mathrm{b}}$ experience the least amount of shift, which can be due to the fact that part of the piperazine ring may be present exterior the cavity (Figure 2). These results are in agreement with FTIR results of inclusion complexes [6] [7] [8] [9]. Chemical shift changes $(\Delta \delta)$ were calculated according to the formula: $\Delta \delta=\delta$ (complex) $-\delta$ (free) (Table 1$)$. 
Table 1. HNMR chemical shifts for $\alpha$-CD and its inclusion complex with OFL.

\begin{tabular}{|c|c|c|c|c|c|c|}
\hline Compound & $\mathrm{H}-1$ & $\mathrm{H}-2$ & $\mathrm{H}-3$ & $\mathrm{H}-4$ & H-5 & H-6 \\
\hline$\alpha-\mathrm{CD}$ & 5.226 & 3.772 & 4.176 & 3.658 & 3.980 & 4.937 \\
\hline $\begin{array}{c}\alpha \text {-CD/OFL } \\
\text { Before degradation }\end{array}$ & 4.921 & 3.452 & 3.855 & 3.491 & 3.569 & 4.698 \\
\hline$\Delta \delta(\mathrm{ppm})$ & 0.299 & 0.320 & 0.321 & 0.167 & 0.411 & 0.239 \\
\hline $\begin{array}{c}\alpha \text {-CD/OFL } \\
\text { After degradation }\end{array}$ & 4.951 & 3.483 & 3.866 & 3.316 & 3.542 & 4.689 \\
\hline$\Delta \delta($ ppm $)$ & 0.275 & 0.289 & 0.310 & 0.342 & 0.438 & 0.248 \\
\hline $\begin{array}{c}\alpha-\mathrm{CD} / \mathrm{OFL} / \mathrm{PEG} \\
\text { Before degradation }\end{array}$ & 4.770 & 3.284 & 3.514 & 2.643 & 3.314 & 4.389 \\
\hline$\Delta \delta(\mathrm{ppm})$ & 0.456 & 0.438 & 0.662 & 0.615 & 0.666 & 0.548 \\
\hline $\begin{array}{c}\alpha \text {-CD/OFL/PEG } \\
\text { After degradation }\end{array}$ & 4.761 & 2.936 & 3.330 & 2.4719 & 3.253 & 4.565 \\
\hline$\Delta \delta(\mathrm{ppm})$ & 0.465 & 0.836 & 0.846 & 0.639 & 0.727 & 0.372 \\
\hline
\end{tabular}

The observed chemical shifts of H-3 and H-5 of $\alpha$-CD host and generating new chemical shifts and splitting with respect to pure compounds upon complexation indicate that the complexes were obtained. Also, results indicated that UV light didn't effect on the structure of OFL in the form of inclusion complex (Figure 2).

The stoichiometries of the complexes of OFL with $\alpha-\mathrm{CD}$ were determined using the continuous variation method (Job's method). Various researches were published in the case of formation of inclusion complex between organic guest molecules such as OFL and other floxacin families with CD in DMSO [11] [12].

For this reason, the guest and the host molecules were distributed among nine NMR tubes, varying the molar ratio from 1:9 to 9:1 at a constant total concentration of $2 \mathrm{mM}$. The complexation-induced shifts of $\mathrm{H}-5$ of $\alpha$-CD are shown on the Job plot where the apex at 0.5 indicates the formation of a 1:1 complex between $\alpha$-CD and OFL which was previously obtained by stoichiometry's analysis (Figure 3).

\subsection{FTIR Results of OFL Inclusion Complexes}

The $\alpha$-CD was able to entrap the OFL drug inside its cavity by forming an inclusion complex and prevent the effects of environmental factors on the drug. FTIR of $\alpha$-CD, OFL and their inclusion complexes is shown in Figure 4 and Table 2. FTIR peaks of $\alpha$-CD were observed at $3369 \mathrm{~cm}^{-1}, 2960 \mathrm{~cm}^{-1}, 1290 \mathrm{~cm}^{-1}$, and 1069 $\mathrm{cm}^{-1}$ which corresponds to the symmetric and antisymmetric stretching of $v(\mathrm{OH}), v\left(\mathrm{CH}_{2}\right), v(\mathrm{C}-\mathrm{C})$ and bending vibration of $v(\mathrm{O}-\mathrm{H})$ respectively.

As for PEG, characteristic peaks are seen at $3400 \mathrm{~cm}^{-1}, 2900 \mathrm{~cm}^{-1}, 1450-1292$ $\mathrm{cm}^{1}, 1250 \mathrm{~cm}^{-1}, 1100-1060 \mathrm{~cm}^{-1}$, in turn, can be attributed to O-H stretching of the hydroxyl group, $\mathrm{C}-\mathrm{H}$ stretches of alkanes, $\mathrm{C}-\mathrm{H}$ scissor and bending, $\mathrm{C}-\mathrm{O}$ stretching of alcohol and C-O-C of ether, respectively. The PEG molecule plays a 


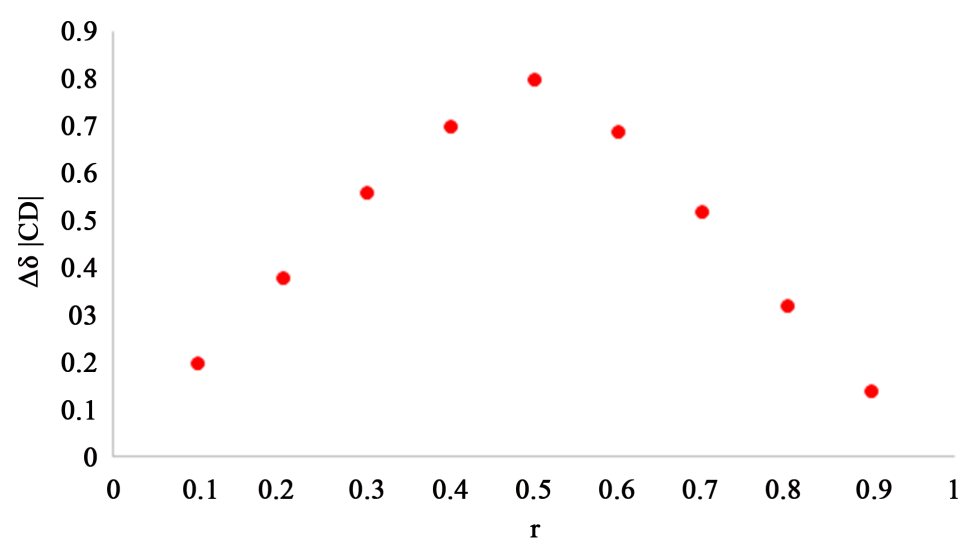

Figure 3. Job's plot using the chemical shift of the H-5 signal of $\alpha$-CD of the complex of daidzein with $\alpha$ - $\mathrm{CD}$, " $\mathrm{r}$ " is the molar fraction of $\alpha$ - $\mathrm{CD}$ in the guest/ $\alpha$-CD mixture.

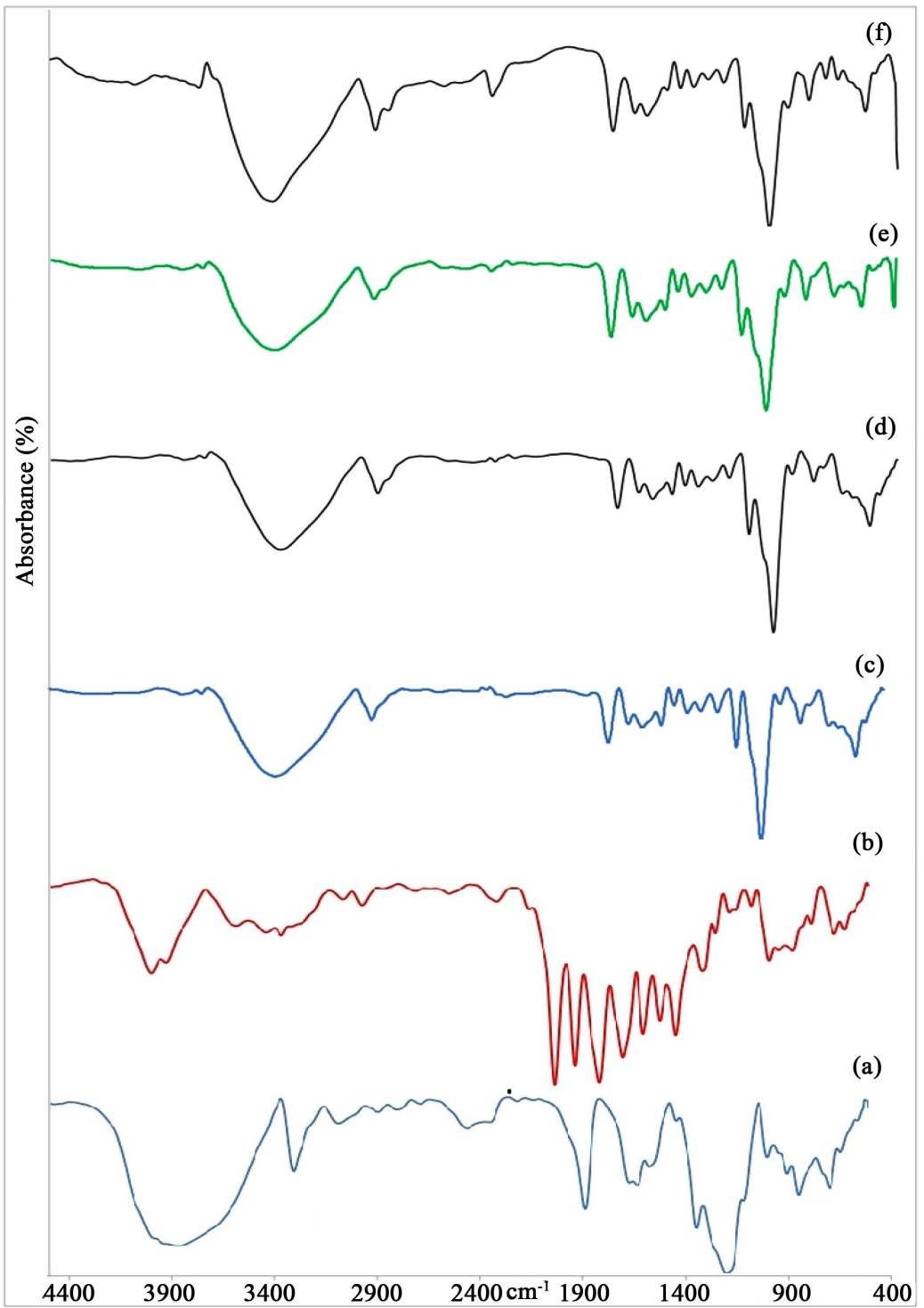

Figure 4. FTIR spectra of (a) $\alpha$-CD; (b) OFL; (c) $\alpha$-CD/OFL and (d) $\alpha$-CD/OFL/PEG before degradation; (e) $\alpha$-CD/OFL and (f) $\alpha$-CD/OFL/PEG after degradation. 
Table 2. FTIR characteristic peaks of OFL.

\begin{tabular}{cc}
\hline Peaks $\left(\mathrm{cm}^{-1}\right)$ & Peak assignment \\
$3300-3200$ & stretching vibration of OH groups \\
$2950-2900$ & Aromatic, cyclic enes Alkyl groups $v=\mathrm{CH} \& \mathrm{Ar}-\mathrm{H}$ \\
2750 & $\mathrm{CH}_{3}$ of Alkyl groups \\
$1900-1500$ & double-bonded functional groups \\
$1750-1700$ & N-H bending vibration of quinolones \\
$1650-1550$ & $\mathrm{CH}_{3}$ and $\mathrm{CH}_{2}$ of Alkyl groups \\
$1580-1500$ & stretching vibration of carbonyl C=O group of acids \\
$1500-1400$ & O-H bending vibration of carboxylic acid \\
$1400-1350$ & stretching vibration of C-N \\
1352,1283 & C-O-C stretching vibration of Oxo group \\
$1250-1200$ & stretching C-F group of Fluorine group \\
$1050-1000$ & $=\mathrm{C}-\mathrm{H}$ out of plane bending vibration of Aromatics \& enes \\
$950-800$ &
\end{tabular}

key role in threading process of CDs molecules. Once the $\alpha$-CD cavity faces the end of the PEG chain, the initial threading occurs, provided the two reactants are mutually oriented in the proper way. The initial threading is favored by the attractive interactions that are established between the $\alpha$-CD internal cavity and the - $\mathrm{O}-\mathrm{CH}_{2}-\mathrm{CH}_{2}$-units of the linear PEG chain. When the polyrotaxane is finally obtained, attractive interactions are established between different particles and produce large aggregates that easily precipitate and form a white thick gel that entraps a significant amount of OFL. So the amount of entrapped OFL increased.

Various groups are present in OFL structure indicating formation of an inclusion complex between $\alpha$-CD/OFL by presence these peaks in FTIR of inclusion complexes, including $\mathrm{C}-\mathrm{O}$ group, $\mathrm{COOH}$ group, $\mathrm{NH}-\mathrm{CH}_{3}$ group and aromatic groups. Some peaks of OFL shifted or disappeared upon entrapment OFL in $\alpha$-CD cavity, once inclusion complex was formed between OFL and $\alpha$-CD, hydroxyl groups of $\alpha$-CD formed interaction by OFL groups via hydrogen bonding formation, hence $\mathrm{O}-\mathrm{H}$ stretching peaks were shifted to higher frequency and became narrower than pure $\alpha$-CD.

\subsection{XRD Results}

As can be seen, Figure 5. Illustrate XRD peaks of pure $\alpha$-CD, OFL and their inclusion complexes before and after degradation by UV. Appearing of new peaks or shifting the peaks to a new position which is closely tied to change crystalline or amorphous of guest molecules indicates formation of an inclusion complex between CDs and guest molecules. Characteristic peaks of OFL are seen at $2 \theta=$ 8, 12 - 14, 15, 17, 18 - 21, 22 [6] [7] [8] [9]. $\alpha$-CD displays characteristic peaks 


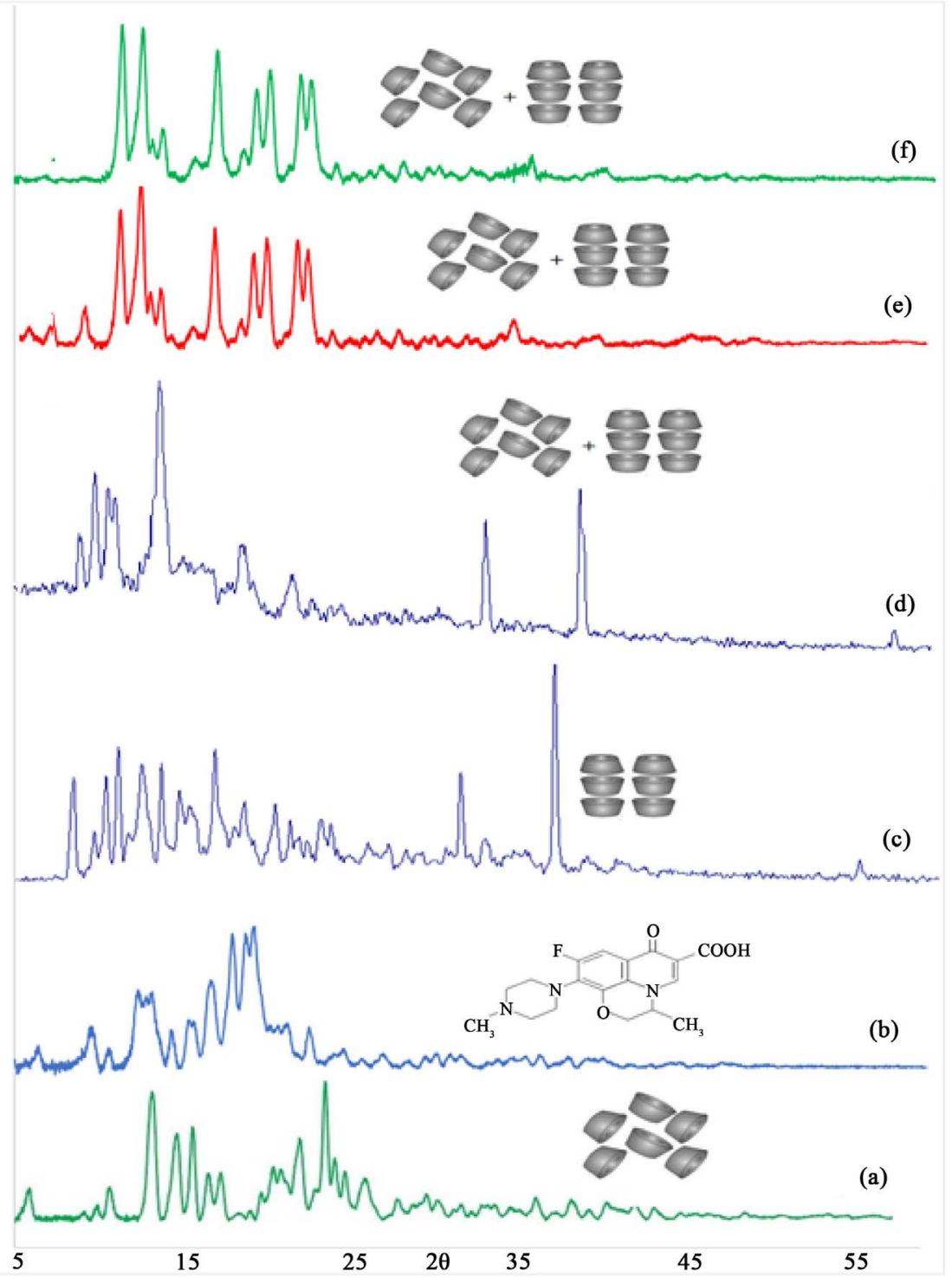

Figure 5. XRD images of (a) pure $\alpha$-CD; (b) pure OFL; (c) $\alpha$-CD/OFL and (d) $\alpha$-CD/OFL/PEG before degradation; (e) $\alpha$-CD/OFL and (f) $\alpha$-CD/OFL/PEG after degradation.

at $2 \theta=7.5,11,13,14,18,20,22,25$ and $2 \theta=5,8,12,23,30$, which is related to formation of an inclusion complex in the form of channel-type and cage type, respectively. New diffraction peaks are appearing which indicated the formation of complex between $\alpha$-CD and OFL as a mixture of the cage and channel type crystals under formation of an inclusion complex between $\alpha$-CD/OFL at two conditions. On the other hand, by insertion of the guest molecule in the $\alpha$-CD cavity, pure $\alpha$-CD and OFL characteristic peaks were not recognized and new peaks appeared, hence inclusion complexes are characterized with various crystalline structures than initial materials. PEG4000 powder showed sharp diffraction peaks at 23.5, 25 and 29 implying obvious crystallinities [6] [7] [8] [9].

The XRD results confirmed that the inclusion complexes formed are crystal- 
line. Results showed that under sonic energy inclusion complexes of CDs are head-to-head channel-type and CDs molecules are stacked along an OFL axis to form a cylinder. Sonic energy showed a major effect on the formation of discrete size crystals with channel-type structure. At room temperature, obtained crystals are a mixture of cage type and channel-type crystals [5] [6] [7]. Results after degradation are same to samples before degradation and the peaks are similar which is related to OFL/CD inclusion complex photostability.

When the drug is exposed to UVA light, the drug peaks remain without any significant change and disappearance, and are only slightly decreased, indicating the effect of $\alpha$-CD on the optical stability of the drug. In the presence of $20 \%$ (w/w) PEG polymer, increasing polymer content increased the inhibitory effect of optical degradation of the drug and its integrity are preserved to a high level, so that it could keep the drug peaks before and after the exposure to light almost constant and prevent the reduction in the characteristic peak intensity of the drug [6] [10] [11].

To use the UV device, the standard samples were prepared from pure OFL solution with the concentrations of 2 ppm, 4 ppm, 6 ppm, 8 ppm and 10 ppm, which eventually resulted in two peaks of OFL at $228 \mathrm{~nm}$ and $273 \mathrm{~nm}$ which are considered the characteristic peak of OFL.

Figure 6 shows the peaks from the optical degradation of the drug using UV, showing the degradation peak in $234 \mathrm{~nm}$ and the OFL peak shift from $273 \mathrm{~nm}$ to $272 \mathrm{~nm}$ and from $228 \mathrm{~nm}$ to $222 \mathrm{~nm}$, which can be due to the isomeric structure of OFL. However, none of the prepared complexes show any new peak resulting from the drug degradation which indicated protection of OFL via formation of inclusion complex with $\alpha$-CD. The sharp peak of pure OFL is due to an increase in the concentration of the drug in the pure sample comparing other samples.

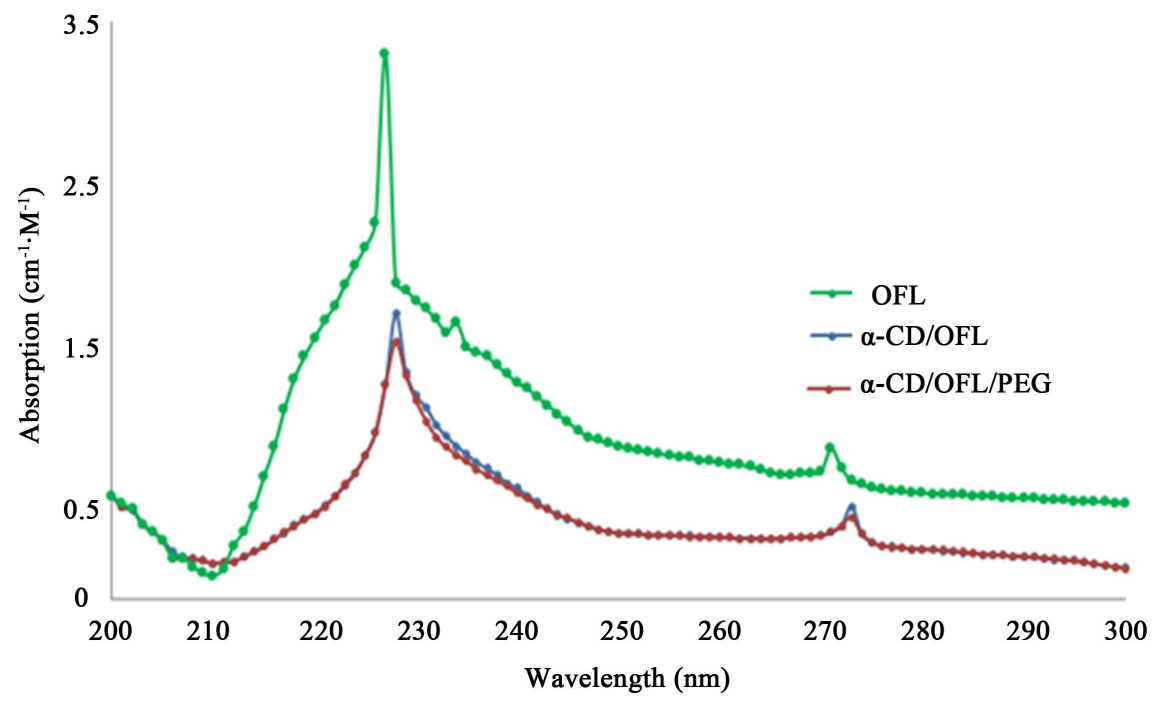

Figure 6. OFL optical degradation and complexes prepared using UV. 


\subsection{PVA-Based Nanofibers Containing Inclusion Complex of CDs/OFL}

The average diameter of obtained nanofibers in various conditions was calculated (Table 3 ) in order to find the optimum condition. The minimum diameter is obtained in $3 \mathrm{ml} / \mathrm{h}$ food rate, $15 \mathrm{kV}$ and distance between the needle tip and the collector (aluminum foil covered collector) was fixed as $20 \mathrm{~cm}$. Electrospinning of all samples was performed at this condition [6] [7] [8] [9].

Incorporation of OFL in PVA nanofibers changes the viscosity and conductivity of PVA solution (Table 4). By increasing viscosity of the solution, diameter of the obtained PVA nanofibers increased directly but diameter of the obtained PVA nanofibers showed inverse correlation with the conductivity of the solution. By adding OFL/CDs complexes to PVA solution, formation of hydrogen bonding between OFL/CDs and PVA, increased solution viscosity so the diameter increased. It seems that the complex between CDs and OFL is smaller than pure OFL by formation of inclusion. It seems that the diameter of the nanofiber is much higher than the pure state with the OFL positioning in the structure of polyvinyl alcohol nanofibers. The smaller diameter of PVA nanofibers containing OFL/CDs is related to better distribution of the OFL/CDs in the fiber structure without accumulation [6] [7] [8] [9] [10].

The presence of citric acid causes the breaking of bonds between the $\alpha$-CD molecules, which reduces the formation and accumulation of $\alpha$-CD crystals and, on the other hand, leads to inter-molecular interactions between $\alpha$ - $\mathrm{CD}$, polyvinyl alcohol and citric acid particles. As a result, the citric acid used in an electrospun solution containing the inclusion complex of $\alpha$-CD and OFL can increase

Table 3. Viscosity, conductivity and average diameter of the obtained nanofibers.

\begin{tabular}{cccc}
\hline \multicolumn{3}{c}{ Electrospinning conditions } & Average diameter $(\mathrm{nm})$ \\
\cline { 1 - 3 } Rate $(\mathrm{ml} / \mathrm{h})$ & $\mathrm{d}(\mathrm{cm})$ & Voltage $(\mathrm{V})$ & 374.3 \\
3 & 11 & 15 & 411.6 \\
3 & 11 & 18 & 399.4 \\
3 & 11 & 19 & 398.2 \\
3 & 11 & 14 & 400.6 \\
3 & 16 & 15 & 366.4 \\
3 & 20 & 15 & 420.7 \\
\hline
\end{tabular}

Table 4. Viscosity, conductivity and average diameter of obtained nanofibers.

\begin{tabular}{ccc}
\hline Sample & Viscosity $(\mathrm{mpa} / \mathrm{s})$ & Conductivity $(\mu \mathrm{s} / \mathrm{cm})$ \\
\hline PVA & 291.43 & 7.26 \\
$\mathrm{PVA} / \alpha-\mathrm{CD} / \mathrm{OFL}$ & 315.45 & 4.18 \\
$\mathrm{PVA} / \alpha-\mathrm{CD} / \mathrm{OFL} / \mathrm{PEG}$ & 295.8 & 3.99
\end{tabular}


the viscosity of the solution due to the increased entanglement between the chains. Also, the presence of $\alpha$-CDin an electrospun solution caused the decrease in the solution conductivity due to the interactions between the molecules [6] [7] [8] [9] [10]. The presence of an inclusion complex between the matrix phases decreases the conductivity of the polymer solution. The conductivity of the polymer solution in the presence of citric acid is shown in Table 5 [11] [12] [13] [14].

The SEM images of pure PVA nanofibers, PVA/CIT, obtained PVA/CIT nanofibers containing inclusion complex of $\alpha$-CD/OFL are shown in Figure 5, which indicated formation of nanofibers without beads. The diameter of the fibers ranges from 120 to $300 \mathrm{~nm}$. Figure 5 showed that the nanofibers containing the inclusion complex of drug are well electrospun with $\alpha$-CD without any bead in the structure lacking texture and with a smooth surface. Due to the size of the complexed nanoparticles, it can be well imagined that the formed complexes are placed inside the fiber without creating a distinctive crystal structure. Placing the complexes containing OFL and $\alpha$-CD in the nanofiber structure, due to the interaction of the hydroxyl groups of exterior $\alpha$-CD with PVA and CIT, decreases the conductivity and increases the viscosity of the electrospun solution, which can consequently affected on the diameter of the obtained fibers [6] [7] [8] [9] [12] [13] [14] [15]. EDX images of nanofibers showed the presence of fluorine and nitrogen peaks from the OFL presence in nanofibers could be demonstrated. The presence of PVA as a polymer matrix led to a significant reduction in the fluorine content in the EDX test comparing the SEM images obtained from pure $\mathrm{OFL} / \alpha$-CD complexes, which can be due to the formation of inclusion complexes inside the polymer matrix phase. However, these two elements are not observed in the pure polyvinyl alcohol (Figure 7).

\subsection{Release Profile of PVA Nanofibers Containing OFL/ $\alpha$-CD}

The swelling ratio of nanofibers plays an essential role in the release rate of the drug inside the nanofibers. The polymer swelling capacity is checked by the amount of water it can absorb. Nanofibers welling is one of the basic parameters, because the water that penetrates into the fiber can dissolve the encapsulated drug and convert it from solid to liquid phase, which accelerates the penetration of the drug into the biological membranes of body [6] [7] [8] [9].

Table 5. Conductivity of polymer solution in the presence of citric acid and obtained PVA nanofibers containing nanocontainers.

\begin{tabular}{cc}
\hline Solution & Conductivity $(\boldsymbol{\mu} \mathbf{s} / \mathrm{cm})$ \\
\hline PVA\%9 & 949 \\
PVA\%9 - CIT\%10 & $4.17 \times 10^{3}$ \\
D-1/PVA\%9 - CIT\%10 & 4.11 \\
D-2/PVA\%9 - CIT\%10 & 4.08 \\
\hline
\end{tabular}



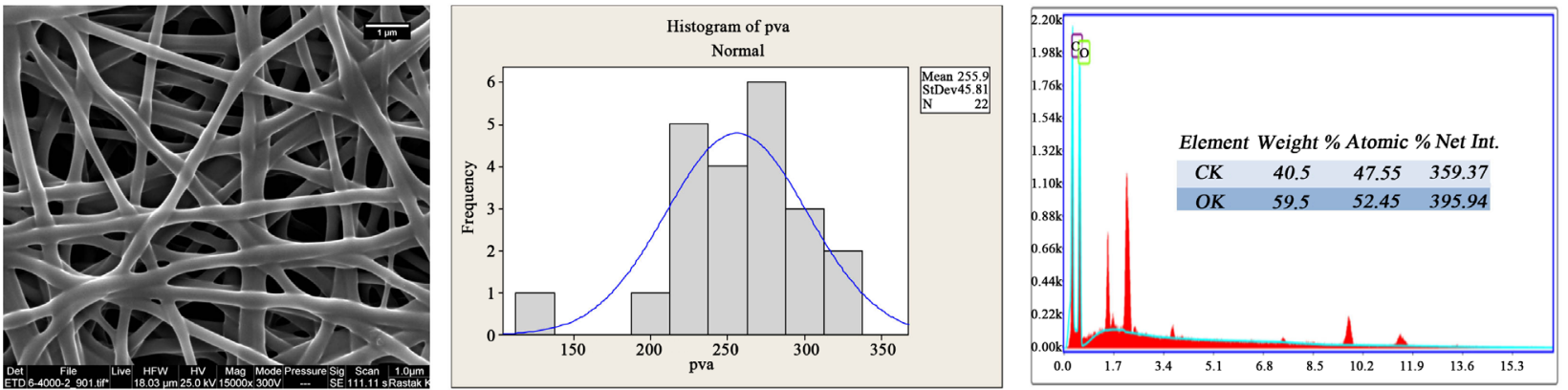

(a)
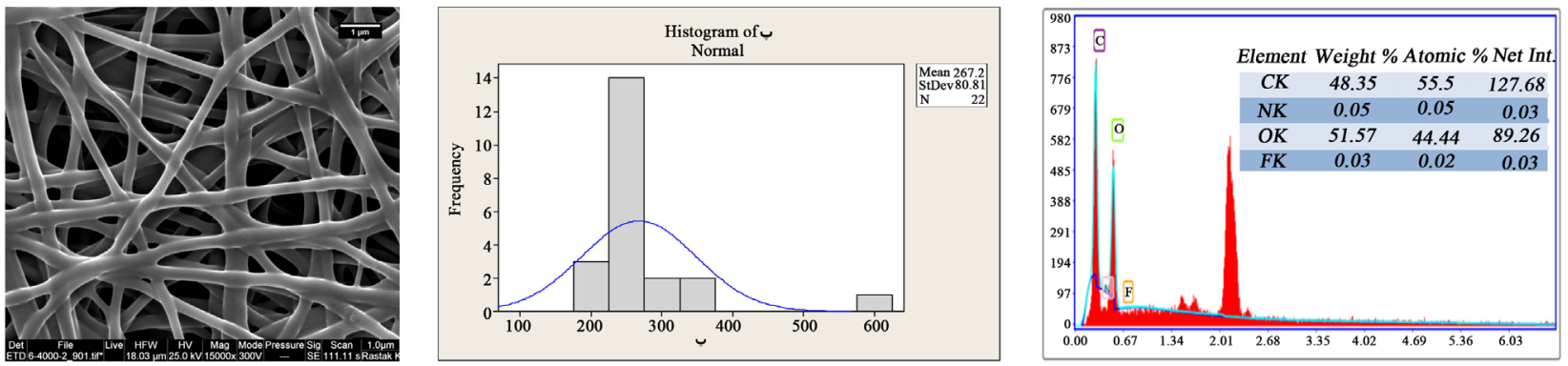

(b)
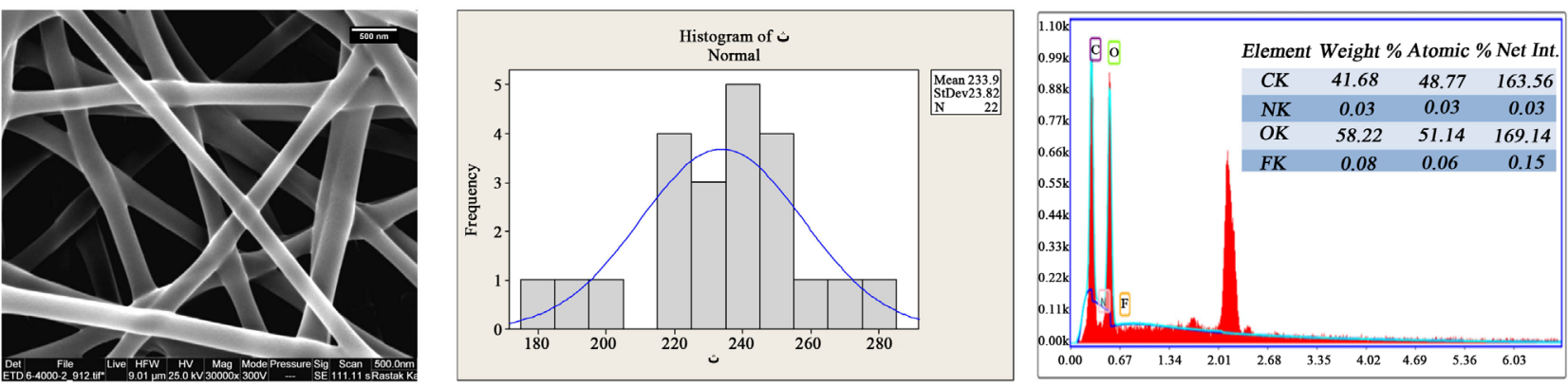

(c)

Figure 7. SEM and EDX images of (a) PVA nanofibers; (b) PVA/ $\alpha$-CD/OFL and (c) PVA/ $\alpha$-CD/OFL/PEG 20\% (w/w) in ultrasonic bath.

To test the stability of the nanofibers in aqueous medium and to measure the release rate of the drug, the release test was performed using nanofibers. As shown in Figure 8, the presence of a polymer matrix also had a significant effect on the amount of drug release during the test period. The rate of release from nanofibers suggests that polyvinyl alcohol has a good cross-linking to CIT and $\alpha$-CD and shows good stability in the phosphate buffer medium, so that after 23 hours from the test, it continues to keep its consistency in the medium and shows insolubility in the aqueous medium. In the early hours of the study, the further release of drugs from nanofibers is seen, which resulted from high inflation levels of the polyvinyl alcohol fibers at the beginning of the experiment, which is gradually increased over time to a constant rate of inflation, and eventually, the release rate of the drug is reduced and reached to a controlled, almost constant level. The high concentration of PVA nanofiber containing OFL results from the high dosage of the drug [6] [7] [8] [9] [11] [16]. In the presence of PEG 


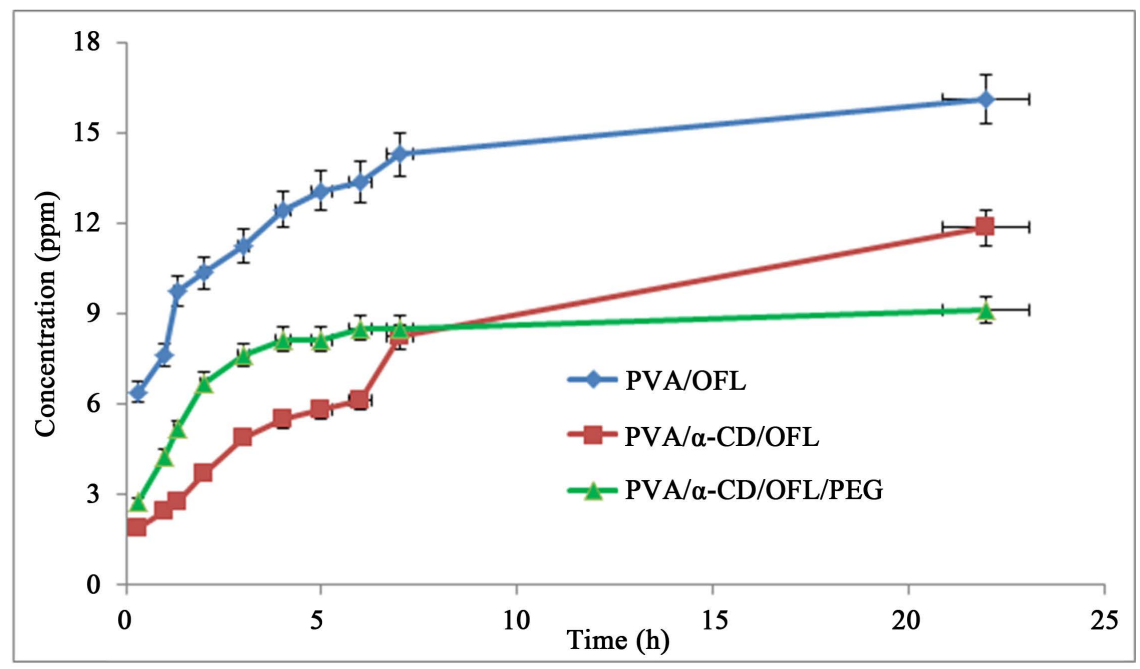

Figure 8. Release of OFL from PVA nanofibers containing in a phosphate buffer at $\mathrm{pH}=$ 7.4 .

higher amount of $\mathrm{OH}$ groups lead to higher solubility of OFL which are interacted with PEG and didn't entrap in CD cavity, after dissolved these OFL groups, the entrapped OFL in CD cavity tend to release and reach to a stable rate.

\subsection{Antibacterial Test}

The antibacterial activity of obtained nanofibers investigated by killing a wide range of Gram-negative and Gram-positive bacteria, which showed by diameters zones of inhibition. Zones of inhibition (including the diameter of disk) on the agar surface around the disks revealed the sensitivity of the microbe to the antibiotic, the rate of diffusion of the antibiotic through the agar and the depth of the agar the sensitivities of the microorganism species and values $<8 \mathrm{~mm}$ were considered as not active against microorganisms. Zone of inhibition is negligible for microorganisms that are resistant to an antibiotic (growing right up to the disk itself) or display a relatively [6] [7] [8] [9].

In this study, anti-bacterial activities of the obtained nanofibers were investigated by killing a wide range of Gram-negative and Gram-positive bacteria. For this purpose, Escherichia coli (E. Coli; as a Gram-negative bacteria) and Staphylococcus aureus ( $S$. aureus, as a Gram-positive bacteria) were used (Figure 9 and Table 6). This test investigated the effect of OFL on the growth of various bacteria. In all samples, the fibers are prepared on an aluminum foil of $1 \times 1 \mathrm{~cm}^{3}$. OFL has the ability to prevent the growth of gram-negative and gram-positive bacteria due to the inhibition of DNA gyrase and prevent bacterial replication. As shown in Figure 7, the OFL/PVA showed significant antibacterial activity based on halo growth due to the high dosage of the drug in this sample. In all cases, the growth of the halo was observed, which is related to lower number of $\mathrm{OH}$ groups of medium which controlled solubility of OFL in aqueous solution and the antibacterial effect is remain a long time. But in the presence of $\alpha$-CD, 
E. Coli
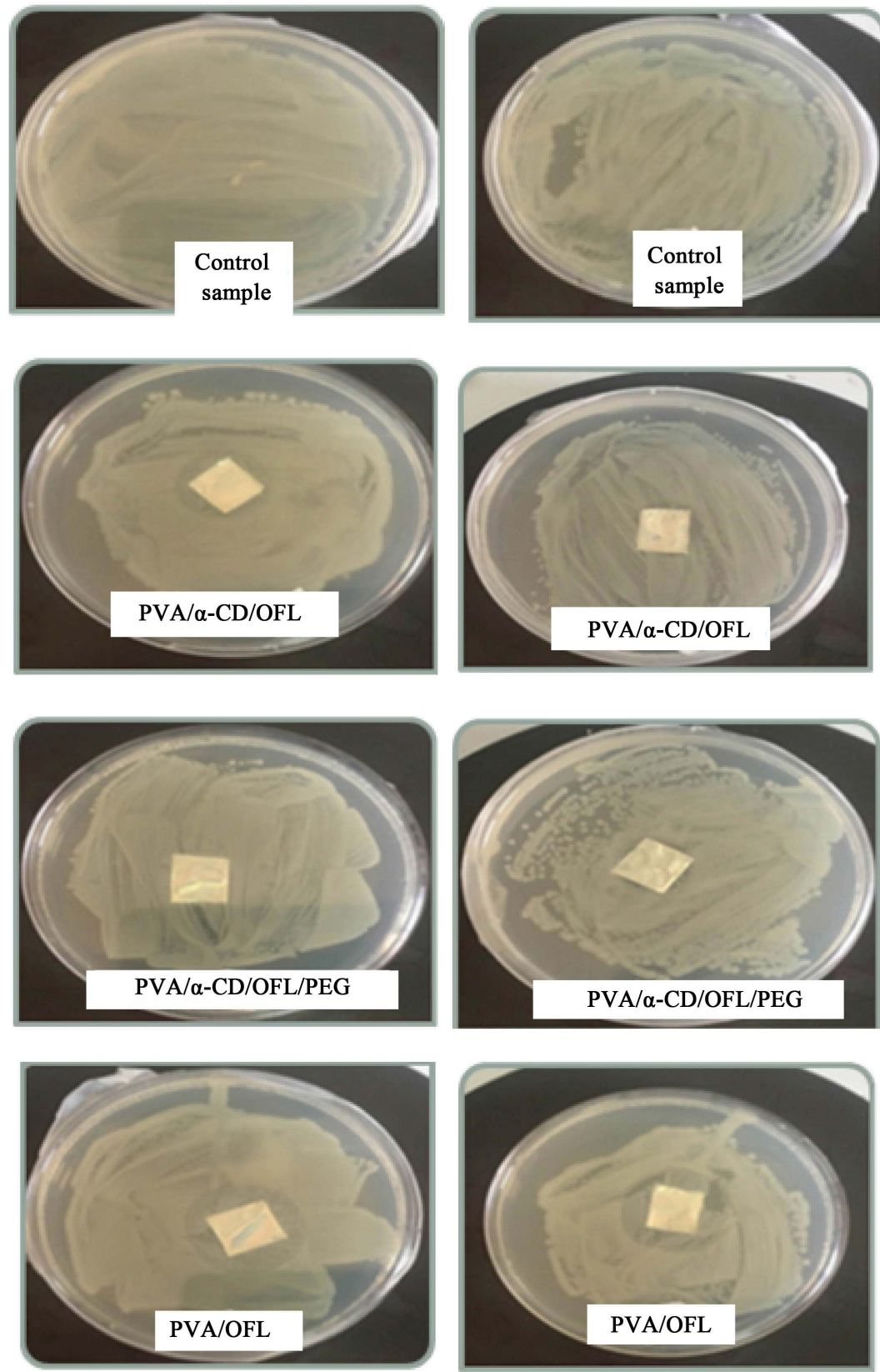

Figure 9. Antibacterial test of obtained nanofibers in the presence of E. Coli and S. Aureus bacteria.

Table 6. Diameter of inhibition zone of PVA nanofibers containing obtained $\alpha$-CD/OFL complexes.

\begin{tabular}{ccc}
\hline \multirow{2}{*}{ Sample } & \multicolumn{2}{c}{ Diameter of inhibition zone } \\
\cline { 2 - 3 } & E. coli $\left(1.5 \times 10^{7}\right)$ & S. aureus $\left(1.5 \times 10^{7}\right)$ \\
\hline PVA & No aura & No aura \\
PVA/OFL & $23 \mathrm{~mm}$ & $20 \mathrm{~mm}$ \\
$\alpha$-CD/OFL & $13 \mathrm{~mm}$ & $11 \mathrm{~mm}$ \\
$\alpha$-CD/OFL/PEG & $16 \mathrm{~mm}$ & $10 \mathrm{~mm}$ \\
\hline
\end{tabular}


higher number of hydroxyl groups form $\alpha$-CD lead to higher solubility of OFL and antibacterial effect remains short times.

\section{Conclusion}

Formation of inclusion complex between $\alpha$-CD and OFL in ultrasonic bath was studied by ${ }^{1} \mathrm{H}-\mathrm{NMR}$ and FTIR and formation of the inclusion complex was determined. Chemical shifts in ${ }^{1} \mathrm{H}$-NMR indicated insertion of OFL in $\alpha$-CD cavity and formation of inclusion complex via hydrogen bonding. Photodegradation of $\alpha$-CD/OFL was studied by FTIR which confirmed stability of OFL. Phase solubility studies with UV confirmed solubility and drug release form $\alpha$-CD/OFL. Presence of PEG improved complex formation, water solubility, and this, in turn, can be attributed to increased hydroxyl groups in the reaction medium and increase the formation of complexes between OFL and hydroxyl groups. By incorporation, $\mathrm{OFL} / \mathrm{CDs}$ in PVA nanofibers viscosity increased which is related to formation of hydrogen bonding between PVA and CDs. PVA nanofibers containing OFL and $\alpha$-CD/OFL inclusion complexes were investigated which showed high antibacterial activity with a controlled release of OFL from nanofibers.

\section{Conflicts of Interest}

The authors declare no conflicts of interest regarding the publication of this paper.

\section{References}

[1] Yamaguchi, J., Oguchi, H., Tokudome, Y. and Katsuyama, M. (1994) A Case of Photosensitive Drug Eruption Induced by Sparfloxacin. Nishinihon Journal of Dermatology, 56, 1146-1149. https://doi.org/10.2336/nishinihonhifu.56.1146

[2] Zhanel, G.G., Ennis, K., Vercaigne, L., Walkty, A., Gin, A.S., Embil, J., Smith, H. and Hoban, D.J. (2002) A Critical Review of the Fluoroquinolones, Focus on Respiratory Tract Infections. Drugs, 62, 13-59. https://doi.org/10.2165/00003495-200262010-00002

[3] Tiefenbacher, E.M., Haen, E., Przybilla, B. and Kurz, H. (1994) Photodegradation of Some Quinolones Used as Antimicrobial Therapeutics. Journal of Pharmaceutical Sciences, 83, 463-467. https://doi.org/10.1002/jps.2600830403

[4] World Health Organization (2009) Annex: Stability Testing of Active Pharmaceutical Ingredients and Finished Pharmaceutical Products. WHO Technical Report Series, Vol. 953.

[5] Amiri, S. and Amiri, S. (2017) Cyclodextrins: Properties and Industrial Applications. John Wiley \& Sons Ltd., Hoboken, NJ. https://doi.org/10.1002/9781119247609

[6] Amiri, S. and Nalbandi, B. (2018) Improve Solubility and Bioavailability of Silver Sulfadiazine via Formation of Inclusion Complex by Cyclodextrin. Journal of Inorganic and Organometallic Polymers and Materials, 28, 1764-1774. https://doi.org/10.1007/s10904-018-0809-8

[7] Masoumi, S., Amiri, S. and Bahrami, S.H. (2017) PCL-Based Nanofibers Loaded 
with Ciprofloxacin/Cyclodextrin Containers. Journal of the Textile Institute, 109, 1044-1053. https://doi.org/10.1080/00405000.2017.1398625

[8] Masoumi, S., Amiri, S. and Bahrami, S.H. (2018) PCL-Based Nanofibers Containing Ibuprofen/Cyclodextrins Nanocontainers: A Potential Candidate for Drug Delivery Application. Journal of Industrial Textiles, 48, 1420-1438. https://doi.org/10.1177/1528083718764910

[9] Amiri, S. and Rahimi, A. (2018) Poly( $\varepsilon$-Caprolactone) Electrospun Nanofibers Containing Cinnamon Essential Oil Nanocapsules: A Promising Technique for Controlled Release and High Solubility. Journal of Industrial Textiles, 48, 1527-1544. https://doi.org/10.1177/1528083718764911

[10] Rajendiran, N., Mohandoss, T. and Thulasidhasan, J. (2015) Encapsulation of Ciprofloxacin, Sparfloxacin, and Ofloxacin Drugs with $\alpha$ - and $\beta$-Cyclodextrins: Spectral and Molecular Modelling Studies. Physics and Chemistry of Liquids, 54, 193-212. https://doi.org/10.1080/00319104.2015.1074046

[11] Sherinivas, K. and Udupa, N. (1996) Physicochemical Study of Ciprofloxacin with $\beta$-Cyclodextrin. Pharmaceutical Sciences, 2, 451455.

[12] Al-Rawashdeh, N., Shawakfeh, K. and Ata, S. (2008) Photostability Study of Ciprofloxacin with Cyclodextrins. PACCON2008 (Pure and Applied Chemistry International Conference), 30 January-1 February 2008, Sofitel Centara Grand, Bangkok, Thailand, 416-421.

[13] Padhan, P., Sethy, A. and Behera, P.K. (2017) Host-Guest Interaction between Ofloxacin-b-Cyclodextrin Complexes in Acidic and Neutral $\mathrm{pH}$, A Fluorescence Quenching Study. Journal of Photochemistry and Photobiology A: Chemistry, 337, 165-171.

[14] Amiri, S. and Rahimi, A. (2018) Poly( $\varepsilon$-Caprolactone) Electrospun Nanofibers Containing CEO Containers: Enhanced Solubility, Dissolution and Physical Stability of CUR via Formation of Inclusion Complex with Cyclodextrins. Journal of Industrial Textiles, 68, 669-679. https://doi.org/10.1080/00914037.2018.1482467

[15] Heidari, M., Bahrami, H. and Ranjbar-Mohammadi, M. (2017) Fabrication, Optimization and Characterization of Electrospun Poly(Caprolactone)/Gelatin/Graphene Nanofibrous Mats. Materials Science and Engineering. C, 78, 218-229.

[16] Fuat, T. and Tamer, U. (2019) Electrospinning of Cyclodextrin Functional Nanofibers for Drug Delivery Applications. Pharmaceutics, 11, 35 p. 